\title{
Intrinsic non-uniqueness of the acoustic full waveform inverse problem
}

\author{
Chao Lyu ${ }^{1,2}$, Yann Capdeville ${ }^{2}$, David Al-Attar ${ }^{3}$, Liang Zhao ${ }^{1}$ \\ ${ }^{1}$ State Key Laboratory of Lithospheric Evolution, Institute of Geology and Geophysics, \\ Chinese Academy of Sciences, 100029 Beijing, China \\ ${ }^{2}$ CNRS, UMR 6112 Laboratoire de Planétologie et Géodynamique, Université de Nantes, \\ France; email:yann.capdeville@univ-nantes.fr \\ ${ }^{3}$ Bullard Laboratories, Department of Earth Sciences, University of Cambridge, \\ United Kingdom.
}

\section{SUMMARY}

In the context of seismic imaging, full waveform inversion (FWI) is increasingly popular. Because of its lower numerical cost, the acoustic approximation is often used, especially at the exploration geophysics scale, both for tests and for real data. Moreover, some research domains such as helioseismology face true acoustic media for which FWI can be useful. In this work, an argument that combines particle relabelling and homogenization is used to show that the general acoustic inverse problem based on band-limited data is intrinsically non-unique. It follows that the results of such inversions should be interpreted with caution. To illustrate these ideas, we consider 2-D numerical FWI examples based on a Gauss-Newton iterative inversion scheme, and demonstrate effects of this non-uniqueness in the local optimization context.

keywords: Inverse theory; Waveform inversion; Theoretical seismology; Computational seismology; Numerical modelling; Seismic anisotropy. 


\section{INTRODUCTION}

Full Waveform Inversion (FWI) is increasingly used in seismic exploration and seismology to image the Earth interior at a wide range of scales (Virieux \& Operto. 2009). FWI methods are based on a classical data-fitting approach to inverse problems, with the data comprising the observed waveforms. For most applications it is necessary to consider wave propagation in an elastic medium, though acoustic waves are relevant in some areas including helioseismology. Moreover, the acoustic approximation of the elastic problem is numerically cheaper, and is frequently used in exploration geophysics for both tests and real applications.

FWI is numerically very challenging, with global methods based on Monte-Carlo inversion being typically infeasible. Local optimization approaches are, therefore, almost always the only option available. Such local methods can only find one "best" solution while, potentially, allowing for uncertainty quantification in a linearised sense. Moreover, for all practical FWI methods, the properties of seismic waves along with the numerical cost of including higher frequencies means that only a limited frequency band can be used, and this places an upper bound on the spatial resolution obtainable within inversions.

An important question for FWI, and for inverse problems in general, is if the solutions obtained are unique or, speaking more practically, whether a model that fits the data acceptably is necessarily close to the truth? As noted above, the band limited nature of FWI studies places an upper limit on model resolution, while a lack of data coverage is a further source of uncertainty. In principle, however, both these effects can be mitigated against, though at a perhaps substantial cost. It might reasonably be asked whether these are the only sources of uncertainty, or if there is a more fundamental problem that must be confronted. For a very limited range of theoretical cases, the uniqueness of FWI can be mathematically demonstrated, but only in situations with highly-idealised data (e.g. Nakamura \& Uhlmann 1994). In other equally idealised situations, however, FWI can be shown to have generally non-unique solutions. In particular, this is the case for a simple 1-D inversion where it can be shown that only the impedance can be recovered from boundary measurements, while the density-acoustic parameter couple is out of reach (Bamberger et al. 1979). Building on this example, the aim of this article is to explore another potential source of non-uniqueness within FWI based on the idea of "particle relabelling" which may be seen as a higher-dimensional generalization of Bamberger et al. 1979's work.

Before going further, we need to say a few words about the elastic and acoustic wave equations and the mechanical properties involved. For the elastic wave equation, the constitutive relation linearly linking the stress and the strain involves the elastic tensor $\boldsymbol{c}$, a fourth-order 
tensor (Aki \& Richards 1980). It has a major $\left(c_{i j k l}=c_{k l i j}\right)$ and minor $\left(c_{i j k l}=c_{j i k l}=c_{i j l k}\right)$ symmetries, reducing its number of independent parameters from 81 to 21 in 3-D. Further symmetries can reduce this number of parameters. For example, an isotropic medium implies only 2 independent parameters. The acoustic wave equation can be solved using the pressure formulation (Komatitsch et al. 2000). In that case, the constitutive relation links the velocity with the pressure gradient through the inverse of the density. This is for classical isotropic fluids. This formulation can be extended to anisotropic fluids with the introduction of the inverse density tensor (Cance \& Capdeville 2015). It plays the same role as the elastic tensor but is only an order 2 tensor with one major symmetry. This unusual aspect of the acoustic wave equation is discussed further in this paper.

The particle relabeling method (Al-Attar \& Crawford 2016) shows that the interior properties of an elastic or acoustic medium can be transformed in such a way that the surface waveforms are not changed. This is accomplished by the introduction of a smooth and invertible mapping of the body into itself that leaves the surface fixed. Using this mapping, the original material parameters are transformed in a specific manner such that the surface wavefield remains the same. Particle relabeling is, therefore, an ostensibly strong source of non-uniqueness within FWI. Nevertheless, in the elastic case, it is found that the elastic tensor loses its minor symmetries under such a relabelling transformation. These minor symmetries are physically required, reflecting the invariance of the elastic potential energy under rigid rotations, and hence particle relabelling does not lead to non-uniqueness in the elastic FWI problem since such non-physical models are precluded. The acoustic case is different, however, with the required symmetry of the density tensor being preserved under a relabelling transformation. Nevertheless, if density isotropy can be enforced, this source of non-uniqueness is removed because a relabelling transformation necessarily leads to an anisotropic density.

Homogenization (Capdeville et al. 2010; Capdeville et al. 2020) is a process that determines a smooth effective medium from a rough one such that the limited frequency band waveforms computed in the true and the effective media are the same up to a controlled error. It is a generalization of the Backus long-wavelength equivalent models valid for layered models (Backus 1962). One effect of the homogenization is to introduce apparent anisotropy: the effective medium is in general anisotropic even if the true medium is isotropic. For every FWI, data are used in a limited frequency band: this implies a minimum wavelength and a limited resolution. Because of this limited resolution, the FWI result is always at best the homogenized true model and not the true model (Capdeville \& Métivier 2018). It follows that, for a fine scale true medium, even if it is isotropic, the solution of a FWI is often anisotropic: 
elastic tensor anisotropy in the elastic case and density anisotropy in the acoustic case. As a consequence, allowing anisotropy in the parametrization of the FWI is often necessary for both acoustic and elastic media. Thus in the case of acoustic FWI, unless the true medium is smooth (without sub-wavelength structure) and isotropic, the intrinsic non-uniqueness due to particle relabelling cannot be ruled out by insisting the density must be isotropic.

The objective of this work is to show, through examples, that

- an elastic FWI is immune from a potential non-uniqueness due to particle relabelling;

- an acoustic FWI is immune from a potential non-uniqueness due to particle relabelling if isotropy is enforced;

- there is a non-unique solution to an acoustic FWI in general because of particle relabelling and anisotropy induced by homogenization.

The paper is organized as follows: we first detail the theoretical arguments and we then show some numerical examples of the effect of the particle relabelling non-uniqueness.

\section{THEORETICAL ARGUMENTS}

In this section, we develop the arguments leading to the conclusion that an acoustic FWI is in general intrinsically non-unique. To this end, we need to introduce the elastic and the acoustic wave equations, the notions of particle relabelling and of homogenization.

We consider a domain $\boldsymbol{\Omega}$ in which a set of receivers and a set of seismic sources are placed (see Fig. 1). Within $\boldsymbol{\Omega}$ we can consider either elastic or an acoustic wave propagation.

\subsection{The elastic and acoustic wave equations}

In the elastic case, the particle displacement $\mathbf{u}(\mathbf{x}, t)$ with respect to a reference position is governed by the following elastic wave equation,

$\rho \partial_{t t} \mathbf{u}-\nabla \cdot \mathbf{c}: \nabla \mathbf{u}=\mathbf{f}$,

where $\rho(\mathbf{x})$ is the density, $\mathbf{c}(\mathbf{x})$ is the $4^{\text {th }}$ order elastic tensor, $\mathbf{f}$ represents the seismic source, and, using the Einstein repeated indices summation convention, $[\mathbf{c}: \nabla \mathbf{u}]_{i j}=c_{i j k l} \partial_{k} u_{l}$.

In the acoustic case, the velocity potential $q(\mathbf{x}, t)$ is defined such that $\partial_{t} \mathbf{u}=(1 / \rho) \nabla q$, and is governed by the acoustic wave equation,

$\frac{1}{\kappa} \partial_{t t} q-\nabla \cdot \mathbf{L} \cdot \nabla q=g$

where $\kappa(\mathbf{x})$ is the acoustic bulk modulus, $\mathbf{L}(\mathbf{x})$ is the inverse density tensor and $g$ is the acoustic 
source. $q$ can be related to fluid pressure $p$ with $p=\dot{q}$. The matrix form of the inverse density is not common is geophysics and, for a classical isotropic fluid, we have $L_{i j}=\rho^{-1} \delta_{i j}$, where $\delta_{i j}$ is the Kronecker delta. Unlike solids, a fluid cannot be anisotropic at the material fabric scale. Nevertheless, an acoustic wave propagating in a fluid with heterogeneous structures of a much smaller scale than the minimum wavelength can have an anisotropic behavior. For such cases, to model the effective acoustic wave, (2) needs to be introduced. It is a generalization of the classical acoustic wave equation where anisotropy is allowed (Cance \& Capdeville 2015). For example, for a fluid with a 2-D thin horizontally periodic stratified structure, two effective densities are required and we have $L_{x x}=\langle 1 / \rho\rangle, L_{z z}=1 /\langle\rho\rangle$ and $L_{x z}=L_{z x}=0$, where $\langle$.$\rangle is$ the periodic vertical average. It is worth emphasising that, in the acoustic case, the anisotropy is carried by the inverse density tensor while it is associated with the elastic tensor in the elastic problem.

Note that the acoustic anisotropy we are referring to here is very different from the acoustic anisotropy often used in exploration geophysics as introduced by Alkhalifah (2000). The latter is designed such that acoustic waves can have a similar propagation pattern as $\mathrm{P}$-waves in transversely isotropic solid media. Alkhalifah (2000) qualifies his anisotropy as being nonphysical and it can lead to spurious waves, particularly near to the source (Grechka et al. 2004). Nevertheless, it is useful to model elastic P-waves at a low numerical cost. The physical anisotropy of (2) that arises through homogenization is of different nature, and it could not be used to approximate anisotropic P-wave propagation in solids (Cance \& Capdeville 2015).

\subsection{Full Waveform Inversion}

The objective of a Full Waveform Inversion (FWI) is to retrieve some information about the mechanical properties in $\boldsymbol{\Omega}$ using the waveform signal recorded at the receivers from a set of sources. There are many ways to do this and, here, we consider the minimization of the squared differences between data and synthetic seismograms obtained by solving the wave equations (1) or (2). For example, in the elastic case, the objective is to find models $\mathbf{m}=(\rho, \mathbf{c})$ that minimize

$E(\mathbf{m})=\sum_{r, s} \int_{0}^{T}\left[\mathbf{d}_{s}\left(\mathbf{x}_{r}, t\right)-\mathbf{u}\left(\mathbf{x}_{r}, t ; \mathbf{m}\right)\right]^{2} d t$

where $r$ and $s$ are the receivers and sources indices, $\mathbf{d}$ is the recorded data of duration $T$ and $\mathbf{u}$ the displacement obtained by solving (1) using the model parameter $\mathbf{m}$. An important point for our discussion is that, in practice and because of limitations in both data collection and computing power, FWI can only be solved with limited frequency band data and syn- 


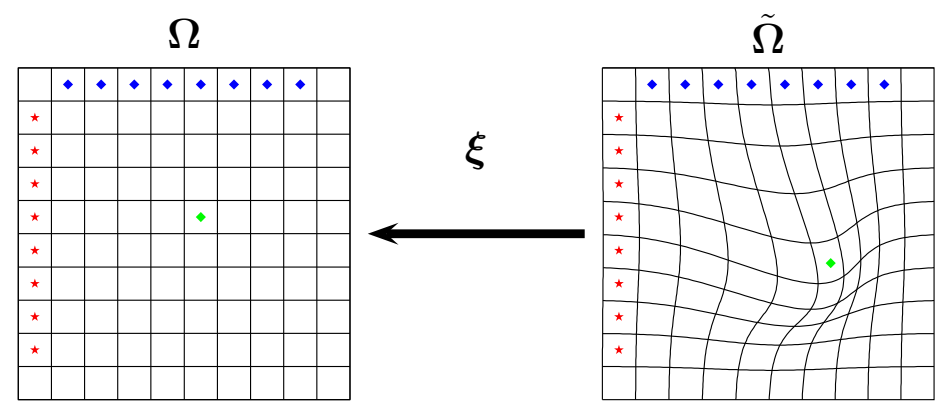

Figure 1. Original domain $(\boldsymbol{\Omega})$ and deformed domain $(\tilde{\boldsymbol{\Omega}})$ for an example of particle relabeling mapping $\boldsymbol{\xi}$. This particular example leaves the position of the sources (red stars) and receivers (blue stars) unchanged.

thetics. This implies that we always have a known maximum signal frequency and, therefore, a wavefield minimum wavelength $\lambda_{\min }$.

\subsection{Particle relabeling transformations}

Building on earlier studies including Woodhouse (1976), Al-Attar \& Crawford (2016) introduced the notion of particle relabeling in seismology. Considering $\boldsymbol{\xi}$, a smooth mapping from $\tilde{\boldsymbol{\Omega}}$ to $\boldsymbol{\Omega}$ with a smooth inverse (see Fig. 1), Al-Attar \& Crawford (2016) showed that:

- in the elastic case, the displacement in the transformed domain is defined by $\tilde{\mathbf{u}}(\mathbf{x}, t)=$ $\mathbf{u}[\boldsymbol{\xi}(\mathbf{x}), t]$ and is the solution of

$\tilde{\rho} \partial_{t t} \tilde{\mathbf{u}}-\tilde{\nabla} \cdot \tilde{\mathbf{c}}: \nabla \tilde{\mathbf{u}}=\tilde{\mathbf{f}}$,

with

$$
\begin{aligned}
\tilde{\rho}(\mathbf{x}) & =J_{\boldsymbol{\xi}}(\mathbf{x}) \rho[\boldsymbol{\xi}(\mathbf{x})] \\
\tilde{c}_{i j k l}(\mathbf{x}) & =J_{\boldsymbol{\xi}}(\mathbf{x})\left[\mathbf{F}_{\boldsymbol{\xi}}(\mathbf{x})^{-1}\right]_{j m}\left[\mathbf{F}_{\boldsymbol{\xi}}(\mathbf{x})^{-1}\right]_{l n} c_{i m k n}[\boldsymbol{\xi}(\mathbf{x})],
\end{aligned}
$$

where $\mathbf{F}_{\boldsymbol{\xi}}=(\nabla \boldsymbol{\xi})^{T}, J_{\boldsymbol{\xi}}=\operatorname{det}\left(\mathbf{F}_{\boldsymbol{\xi}}\right)$, and the summation convention has been applied;

- in the acoustic case, the velocity potential in the transformed domain is defined by $\tilde{q}(\mathbf{x}, t)=q[\boldsymbol{\xi}(\mathbf{x}), t]$, and is the solution of

$\frac{1}{\tilde{\kappa}} \partial_{t t} \tilde{q}-\nabla \cdot \tilde{\mathbf{L}} \cdot \nabla \tilde{q}=\tilde{g}$,

where

$$
\begin{aligned}
\tilde{\kappa}(\mathbf{x}) & =J_{\boldsymbol{\xi}}^{-1}(\mathbf{x}) \kappa[\boldsymbol{\xi}(\mathbf{x})] \\
\tilde{\mathbf{L}}(\mathbf{x}) & =J_{\boldsymbol{\xi}}(\mathbf{x}) \mathbf{F}_{\boldsymbol{\xi}}(\mathbf{x})^{-1} \mathbf{L}[\boldsymbol{\xi}(\mathbf{x})] \mathbf{F}_{\boldsymbol{\xi}}(\mathbf{x})^{-T}
\end{aligned}
$$


An important point within this theory concerns the symmetries of the transformed tensors. The elastic tensor, in the original domain has a major symmetry, $c_{i j k l}=c_{k l i j}$ and two minor symmetries $c_{i j k l}=c_{j i k l}=c_{i j l k}$. Following the transformation however the result $\tilde{\mathbf{c}}$ only possesses the major symmetry while the minor symmetries have been lost. By contrast, the acoustic density tensor is of second-order and has only one major symmetry $L_{i j}=L_{j i}$ that remains after the relabelling transformation is performed.

\subsection{Homogenization}

For a given elastic medium $(\rho, \mathbf{c})$ and a maximum signal frequency $f_{\max }$, it is possible to compute an effective medium $\left(\rho^{*}, \mathbf{c}^{*}\right)$ such that the waveforms computed in $(\rho, \mathbf{c})$ and $\left(\rho^{*}, \mathbf{c}^{*}\right)$ are the same, up to a given accuracy. $\left(\rho^{*}, \mathbf{c}^{*}\right)$ can be obtained thanks to the homogenization operator, $\left(\rho^{*}, \mathbf{c}^{*}\right)=\mathcal{H}^{k_{\max }}(\rho, \mathbf{c})$ where $k_{\max }=1 / \lambda_{\min }$ (Capdeville et al. 2010). This is also true for acoustic media (Cance \& Capdeville 2015). The operator $\mathcal{H}^{k_{\max }}$ is a non-linear operator that, in general, requires the solution of a set of partial differential equations. If the original medium contains small scales, which is very often the case in realistic situations, the effective medium is free of small scales but is not constant: it is just smoother.

For the present work, the homogenization matters for two reasons:

- it can be shown, at least numerically, that the solution of an FWI is, at best, the homogenized effective medium and not the true medium (Capdeville \& Métivier 2018);

- even if the fine scale medium is isotropic, the effective medium is almost always anisotropic (Backus 1962; Capdeville et al. 2010).

To represent such anisotropic effective models in figures, we often choose to compute and plot the nearest isotropic average. In the 2 -D acoustic case it is computed as $1 / \rho^{* \text { iso }}=\left(L_{11}^{*}+\right.$ $\left.L_{22}^{*}\right) / 2$ and the corresponding $V_{P}^{* \text { iso }}=\sqrt{\kappa^{*} / \rho^{* \text { iso }}}$. In the following, we use the notion of total anisotropy, which measure, in the acoustic case for example, the matrix distance between $\mathbf{L}^{*}$ and $\mathbf{L}^{* \text { iso }}$ (Capdeville \& Métivier 2018). The same is also done for the elastic case, using the nearest isotropic elastic tensor $\boldsymbol{c}^{* \text { iso }}$ (see Capdeville \& Métivier (2018) for a more detailed description of such a representation).

\subsection{Consequences of the homogenization and particle relabelling for the uniqueness of the FWI problem}

As discussed in Al-Attar \& Crawford (2016), particle relabelling can be used to demonstrate non-uniqueness for a certain class of inverse problems. Indeed, as shown in Fig. 1, we can find 
non-trivial mapping $\boldsymbol{\xi}$, such that $\boldsymbol{\xi}$ is the identity near the sources and receivers and nontrivial elsewhere. In such cases, the model $(\tilde{\rho}, \tilde{\mathbf{c}})$, respectively $(\tilde{\kappa}, \tilde{\mathbf{L}})$, produced via relabelling would explain the data the same way as the original media $(\rho, \mathbf{c})$, respectively $(\kappa, \mathbf{L})$, does. It is therefore also a solution of an inverse problem based on those data.

In the elastic case, this is not a real problem in practice. Indeed, as we have seen in the previous section, the transformed elastic tensor $\tilde{\mathbf{c}}$ lacks physically required symmetries. When the inverse problem is set up, these physical symmetries are always enforced, and hence the non-uniqueness associated with particle relabelling is eliminated.

In the acoustic case, things can be more problematic because the required symmetry of $\mathbf{L}$ is preserved under a particle relabeling transformation. We might think that, as with the elastic case, this would not be an issue. Indeed, most of the time, only isotropic density acoustic media are inverted. In the isotropic density case, $\mathbf{L}$ is proportional to the identity, but under any non-trivial transformation $\tilde{\mathbf{L}}$ is not. Therefore, forcing an isotropic density removes this source of non-uniqueness within the inverse problem.

This is where homogenization comes in. In the case of FWI, we necessarily work with limited frequency band data. As mentioned in section 2.4, if the real model is not smooth, which is very often the case in practice, then the FWI can, at best, recover an effective homogenized version of the true model. It follows that, even if the true model is isotropic, we almost always need to invert for an anisotropic medium in either the elastic or acoustic cases. As a result, we should not enforce acoustic isotropy and hence must expect acoustic FWI with limited frequency band data to be fundamentally non-unique.

In the next section, we numerically test this idea in a simple 2-D setting.

\section{NUMERICAL EXPERIMENTS}

\subsection{Inversion design}

There are many ways to solve the FWI problem in practice. Here, we choose a simple iterative local optimization scheme: the Gauss-Newton scheme (Pratt et al. 1998). Such methods are very efficient for determining the nearest local minimum to the starting model. They are therefore able to find the global minimum only if it is sufficiently close to the starting model. To parameterize the inversion, to describe the inverted model quantities such as $(\kappa, \mathbf{L})$ for example, we use a simple polynomial per piece expansion: the inverted domain is meshed of square elements and a polynomial expansion is used in each element. As shown by Capdeville \& Métivier 2018, to compare the inverted model and target model, they both need to be 
Table 1. Mechanical properties of the different models used to generate data and their respective effective versions (with a $\left.{ }^{*}\right) . V_{P}^{0}, V_{S}^{0}$ and $\rho^{0}$ are the properties of background model and $\ln \left(V_{P}\right)$, $\delta \ln \left(V_{S}\right)$ and $\delta \ln (\rho)$ the maximum contrast with respect to these background properties. The anisotropy (aniso) is the total anisotropy discussed in Sec. 2.4

\begin{tabular}{|c|c|c|c|c|c|c|c|}
\hline & $V_{P}^{0}$ & $V_{S}^{0}$ & $\rho^{0}$ & $\delta \ln \left(V_{P}\right)$ & $\delta \ln \left(V_{S}\right)$ & $\delta \ln (\rho)$ & aniso \\
\hline models & $\mathrm{km} / \mathrm{s}$ & $\mathrm{km} / \mathrm{s}$ & & $\%$ & $\%$ & & $\%$ \\
\hline Elastic Gaussian & 5.7 & 3.2 & 2.6 & {$[-33 ; 50]$} & $-[29 ; 40]$ & {$[-20 ; 25]$} & 0. \\
\hline Elastic Gaussian* & 5.7 & 3.2 & 2.6 & {$[-33 ; 50]$} & {$[-29 ; 40]$} & {$[-20 ; 25]$} & 0. \\
\hline Acoustic Gaussian & 3.2 & 0. & 2.6 & {$[-20 ; 25]$} & 0. & {$[-11 ; 12]$} & 0. \\
\hline Acoustic Gaussian* & 3.2 & 0. & 2.6 & {$[-22 ; 36]$} & 0. & {$[-13 ; 16]$} & 0.1 \\
\hline Faulted Layered & 3.2 & 0. & 2.6 & {$[-19 ; 17]$} & 0. & {$[-5.7 ; 9.6]$} & 0. \\
\hline Faulted Layered* & 3.2 & 0 . & 2.6 & {$[-12.1 ; 1.3]$} & 0. & {$[-7.1 ; 1.6]$} & 6.4 \\
\hline
\end{tabular}

homogenized. To perform the following tests, we generate synthetic data using the spectral element method (SEM, Komatitsch \& Vilotte 1998) in the target model. The data are then inverted also with SEM used to solve the wave equation, but using a different mesh from the one used to generate the data, making sure to avoid an inversion crime. The mesh used to generate the data is fine, unstructured, and honoring the mechanical discontinuities of the target model (if any). The mesh used for the inversion is a simple regular mesh. Even in the smooth target model case, both meshes are different. A more detailed description of this inversion process can be found in Capdeville \& Métivier 2018.

\subsection{Smooth model elastic test}

The first test is done with data generated in a smooth elastic model. The target model is shown in Fig. 2(a) and described in detail in Table 1. Its homogenized version is shown in Fig. 2(b), and, because it is a smooth model, there is little difference with the true model (a). We perform two inversions using two different inhomogeneous starting models (Fig. 2(c) and (f)). The data are generated using vertical point forces at each source location, successively. We use a Ricker wavelet (second derivative of Gaussian) source time function with a maximum frequency (considered as three times the central frequency of the Ricker) such that the wavefield has a $\lambda_{\min }$ minimum wavelength in the background model. In what follows, all lengths have been non-dimensionalised relative to $\lambda_{\min }$. We perform the two inversions using an anisotropic parameterization, inverting for $(\rho, \mathbf{c})$, enforcing the elastic tensor minor symmetries. The nearest isotropic $\mathrm{P}$-wave velocity $V_{P}^{\text {iso }}$ raw results of the FWI are shown in 
Fig. 2(d) and (g) for the two starting models (c) and (f) respectively. They clearly show an imprint of the starting models and of the spatial parameterization mesh (shown in Fig. 2(a)); they compare poorly to the target model. Nevertheless, once homogenized, the two inverted (Fig. 2(e) and (h) respectively) models are indistinguishable from the target homogenized model and are independent of the starting models. No anisotropy is found in the inversion homogenized results (not shown in Fig. 2).

While this result does not prove that such inversions have a unique solution in general, the results are consistent with the theoretical arguments developed above, with, in particular, there being no problems linked to particle relabelling.

\subsection{Smooth model acoustic test}

We now perform a similar test to the previous one but for the acoustic smooth target model shown in Fig. 3(a) and Tab. 1. We perform four inversions, two enforcing isotropy (inverting for $(1 / \kappa, 1 / \rho))$, and two releasing anisotropy (inverting for $(1 / \kappa, \mathbf{L}))$, using two different starting models. The homogenized inverted models are shown in Fig. 3.(e) (i) (l) and (c) for $V_{P}^{\text {iso }}$ and in Fig. 3.(f) (j) (m) and (p) for the corresponding total anisotropy. Each model explains equally well the data. The isotropic inversions have found the correct model, independently of the starting model. Nevertheless, the anisotropic inversions have not and the results depend on the starting model.

These results are consistent with the theory: the acoustic case is not immune against particle relabelling non-uniqueness as soon as anisotropy is possible in the parameterization. Nevertheless, in the case where we know the medium is smooth and hence has no anisotropy once homogenised, it is not a problem as isotropy can safely be explicitly imposed in the parameterization of the inversion.

\subsection{Rough model acoustic test}

Finally, we present a test in a rough acoustic target model (see Fig. 4 and Tab. 1). It is a simple layered medium with a fault going through the layers (Fig. 4(a)). Once homogenized, the medium is strongly anisotropic; the corresponding nearest isotropic $V_{P}^{* i s o}$ and total anisotropy are plotted in Fig. 4(b) and (c) respectively. In this case, it is found that an isotropic inversion leads to a result that, while not so bad visually (Fig. 4(d)), is certainly not as good as should be expected with such favourable data coverage. Indeed, anisotropy is necessary to explain the data Fig. 4(d) and a full convergence of the inverse problem is not possible without it. Moreover, the lack of information about anisotropy is a problem for identifying the presence of 
faulting, with such features being associated with significant anisotropic signatures (Capdeville \& Métivier 2018). Similarly to the previous test, the anisotropic inversion, here starting from a homogeneous model, is carried out. It gives a result very different from the homogenized target model (Fig. 4(e) and (f)). Nevertheless, this strange model explains very well the data. Moreover, if we place a new source and a new receiver outside the inversion area, the traces computed in the homogenized and in the target models match very well (Fig. 5(a)). If, however, the location of the new receiver is inside the inversion area, the traces do not match anymore (Fig. 5(b)). These results can be interpreted with reference to the particle relabelling theory: hidden below this particular inversion there is probably an unknown $\boldsymbol{\xi}$ transformation which is different from the identity only in the inversion area which would map the true model into the recovered one. Therefore, for the receiver (A), the transformation leaves the receiver where it is and the match is very good. For the receiver (B), inside the inversion area, $\boldsymbol{\xi}$ is different from the identity and the receiver is moved: the traces do not match. Nevertheless, there must be a location $\mathbf{x}_{b^{\prime}}=\boldsymbol{\xi}^{-1}\left(\mathbf{x}_{b}\right)$ such that the trace computed in the inverted model for a receiver in $\mathbf{x}_{b^{\prime}}$ match the trace computed in the target model for a receiver computed in $\mathbf{x}_{b}$. It is worth emphasising that the existence of a transformation $\boldsymbol{\xi}$ here is only conjectured, and there might be some other additional source of non-uniqueness. A number of closely related inverse problems have, however, been studied within the mathematics literature, with uniqueness there being established precisely up to the action of such a transformation (Lee \& Uhlmann 1989; Stefanov \& Uhlmann 2005). In the absence of better arguments we think it reasonable to suppose that the same will hold in this case.

Finally, let us mention that the same inversion performed in the elastic case works well as shown in Capdeville \& Métivier (2018) and, as expected, presents no particle relabelling issues.

\section{DISCUSSION}

Our numerical tests confirm that the solution of a general acoustic FWI with limited frequency band data is intrinsically non-unique. There is no cure against such non-uniqueness. Paving the domain with receivers could work but it is in general not an option in 3-D. But is it a problem in practice? Fortunately, rough acoustic domains able to produce effective anisotropy are not very common in nature, and it is probably safe to impose isotropy in most situations. Nevertheless, they are cases where the inverse problem solution non-uniqueness related to particle relabelling should be kept in mind. Acoustic imaging in Helioseismology is one of them. Another potentially problematic situation can be found in the field of exploration 
geophysics where the acoustic approximation is often used to test ideas and FWI schemes. Pushing those tests further with target models containing subwavelengh geological structures could lead to the non-uniqueness problem studied here.

Outside planetary science and geophysics, another potential field of applications concerned by the particle relabelling non-uniqueness is fluid metamaterial design. It has been shown that, with some specific arrangements of subwavelength inclusions embedded in a fluid, it is possible to obtain a metalfuid with an anisotropic dynamic mass (Popa \& Cummer 2009; Gumen et al. 2011). Such metafluids can have unusual properties such as negative refraction index and can be used to design cloaking devices (Cheng et al. 2008). Using FWI in such a metafluid would lead to a strongly non-unique solution, as explained in this paper. One could imagine using this fact to make the acoustic location and imaging of an object embedded in such a metafluid difficult or impossible.

It is worth emphasising that though the elastic and isotropic acoustic FWI problems should not display a non-uniqueness due to particle relabelling, this does not mean that there might not be other sources of non-uniqueness in addition to those related to data coverage and the use of band-limited data. Finally, if for some reasons someone were to try an elastic FWI without enforcing the elastic tensor's minor symmetries, they should be expected that the same non-uniqueness problem would be seen as for the general acoustic case.

\section{CONCLUSION}

We have proposed that particle relabelling can be a strong source of non-uniqueness for a general acoustic FWI based on limited frequency band data and our numerical tests have confirmed this idea, even if we didn't provide a mathematical proof.

\section{ACKNOWLEDGMENTS}

We thank Andrew Curtis and Klaus Mosegaard as well as the editor, Carle Tape, for their positive and constructive reviews. This work has been funded by the National Key R\&D Program of China (Grant No.2017YFC0601206), the ANR HIWAI (ANR-16-CE31-0022-01) and the China Scholarship Council (CSC, No.201804910289, 2018-2020). The computations were performed thanks to the Centre de Calcul Intensif des Pays de la Loire (CCIPL) resources. The authors would like to thank G. Festa for letting us use and modify his 2D spectral element program.

\section{Data availability}


No new data were generated or analysed in support of this research.

\section{REFERENCES}

Aki, K. \& Richards, P., 1980. Quantitative Seismology: Theory and Methods. Freeman, San Francisco.

Al-Attar, D. \& Crawford, O., 2016, 02. Particle relabelling transformations in elastodynamics. Geophys. J. Int. 205(1), 575-593.

Alkhalifah, T., 2000. An acoustic wave equation for anisotropic media. Geophysics, 65, $1239-1250$.

Backus, G., 1962. Long-wave elastic anisotropy produced by horizontal layering. J. Geophys. Res. 67(11), 4427-4440.

Bamberger, A., Chavent, G. \& Lailly, P., 1979. About the stability of the inverse problem in 1-d wave equations - application to the interpretation of seismic profiles. Applied Mathematics and Optimization 5(1), 1-47.

Cance, P. \& Capdeville, Y., 2015. Validity of the acoustic approximation for elastic waves in heterogeneous media. Geophysics 80(4), T161-T173.

Capdeville, Y., Cupillard, P. \& Singh, S., 2020. Chapter six - an introduction to the two-scale homogenization method for seismology. In B. Moseley \& L. Krischer (Eds.), Machine Learning in Geosciences, Volume 61 of Advances in Geophysics, pp. 217 - 306. Elsevier.

Capdeville, Y., Guillot, L. \& Marigo, J. J., 2010. 2D nonperiodic homogenization to upscale elastic media for P-SV waves. Geophys. J. Int., 182, 903-922.

Capdeville, Y. \& Métivier, L., 2018. Elastic full waveform inversion based on the homogenization method: theoretical framework and 2-d numerical illustrations. Geophys. J. Int. 213(2), 1093-1112.

Cheng, Y., Yang, F., Xu, J. Y. \& Liu, X. J., 2008. A multilayer structured acoustic cloak with homogeneous isotropic materials. Applied Physics Letters 92(15), 151913.

Grechka, V., Zhang, L. \& Rector III, J. W., 2004. Shear waves in acoustic anisotropic media. Geophysics 69(2), 576-582.

Gumen, L., Arriaga, J. \& Krokhin, A., 2011. Metafluid with anisotropic dynamic mass. Low Temperature Physics 37(11), 975-978.

Komatitsch, D., Barnes, C. \& Tromp, J., 2000. Wave propagation near a fluid-solid interface: A spectral-element approach. Geophysics 65(2), 623-631.

Komatitsch, D. \& Vilotte, J. P., 1998. The spectral element method: an effective tool to 
simulate the seismic response of 2D and 3D geological structures. Bull. Seism. Soc. Am., 88, 368-392.

Lee, J. M. \& Uhlmann, G., 1989. Determining anisotropic real-analytic conductivities by boundary measurements. Communications on Pure and Applied Mathematics 42(8), 1097-1112.

Nakamura, G. \& Uhlmann, G., 1994. Global uniqueness for an inverse boundary problem arising in elasticity. Inventiones mathematicae 118(1), 457-474.

Popa, B.-I. \& Cummer, S. A., 2009. Design and characterization of broadband acoustic composite metamaterials. Physical review B 80(17), 174303.

Pratt, R., Shin, C. \& Hicks, G., 1998. Gauss-newton and full newton methods in frequency domain seismic waveform inversion. Geophys. J. Int., 133, 341-362.

Stefanov, P. \& Uhlmann, G., 2005. Boundary rigidity and stability for generic simple metrics. Journal of the American Mathematical Society 18(4), 975-1003.

Virieux, J. \& Operto., S., 2009. An overview of full waveform inversion in exploration geophysics. Geophysics 75(6), WCC127-WCC152.

Woodhouse, J., 1976. On rayleigh's principle. Geophysical Journal International 46(1), $11-22$. 
(a)

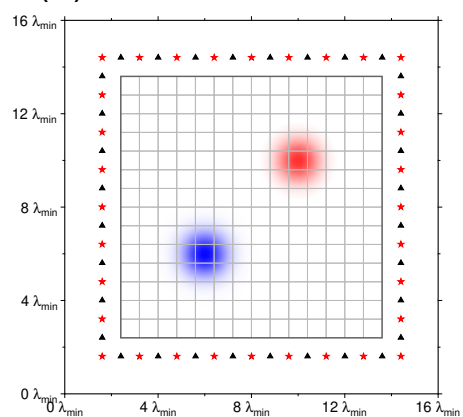

(c)

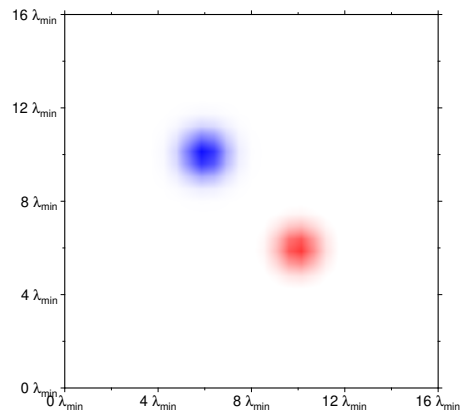

(f)

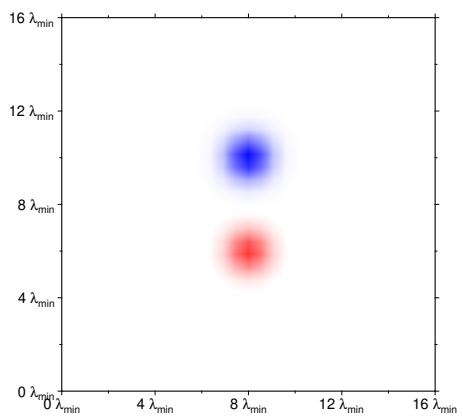

(d)

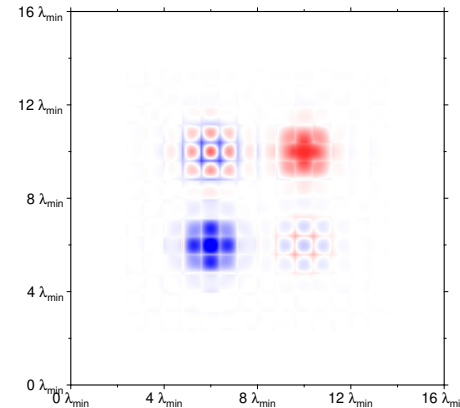

(g)

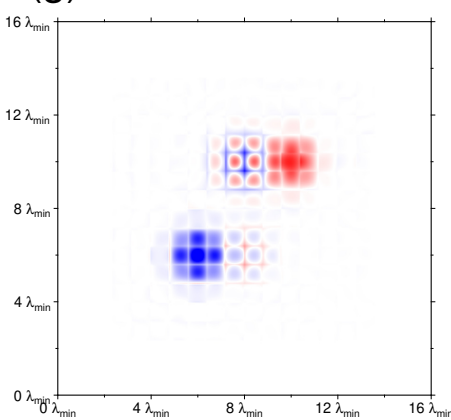

$\delta \ln \mathrm{V}_{\mathrm{P}}(\%)$ (b)

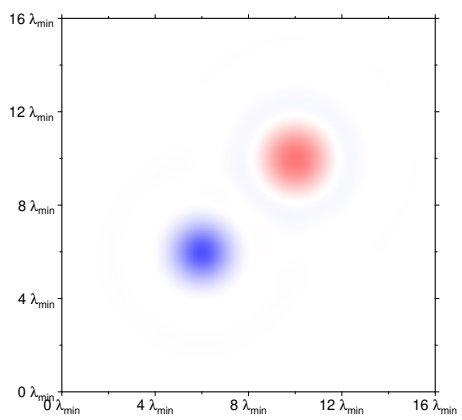

(e)

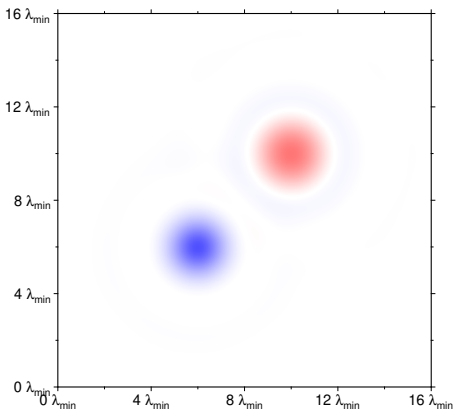

(h)

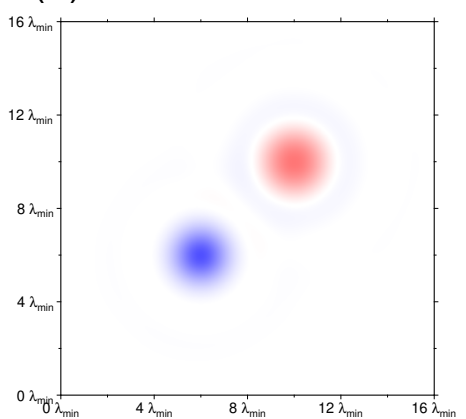

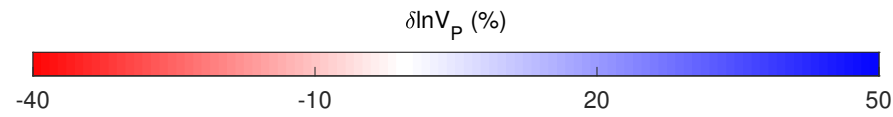

Figure 2. Smooth elastic FWI tests. (a) $P$ wave velocities $\left(V_{P}\right)$ for the target model ; (b) homogenized target model nearest isotropic P-wave velocity $V_{P}^{\text {iso }} ;(\mathrm{c})$ and (f) two starting models $V_{P} ;(\mathrm{d})$ and (g) raw inverted models $V_{P}^{\text {iso }}$ for the starting models (c) and (f) respectively; (e) and (h) homogenized inverted models $V_{P}^{\text {iso }}$ for the starting models (c) and (f) respectively; For each panel, only $V_{P}$ is represented, but the other mechanical properties have the same shape. In (a) the red stars represent the position of the sources and the black triangles represent the position of the receivers. The black square is the area where the model parameters are inverted and the inside mesh is the inversion mesh. 
(a)

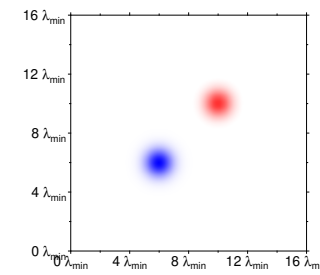

(d)

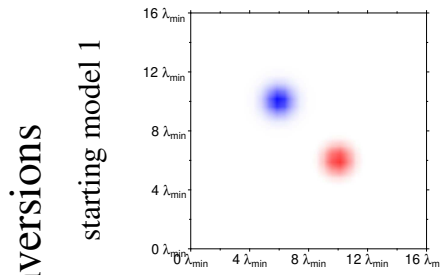

(h)

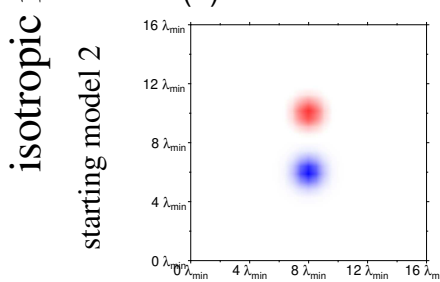

(k)

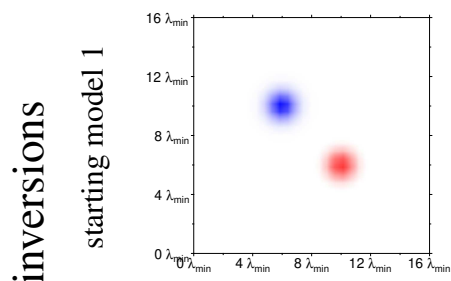

(n)
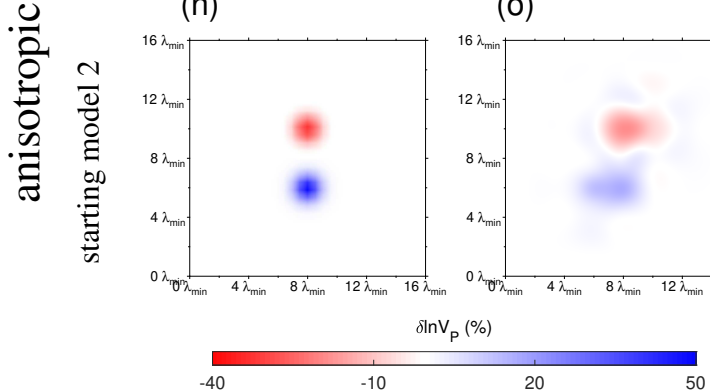

(b)

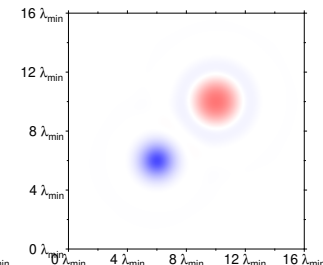

(e)

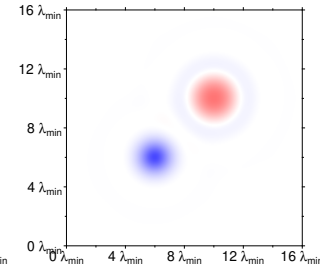

(i)

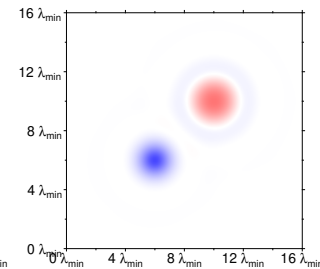

(I)

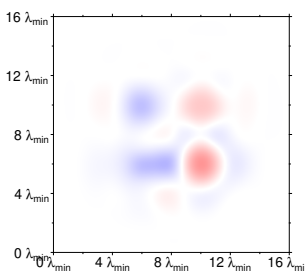

(o)

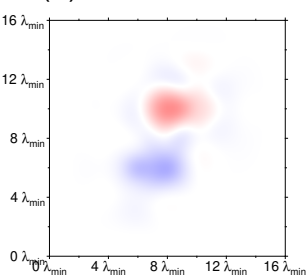

(c)

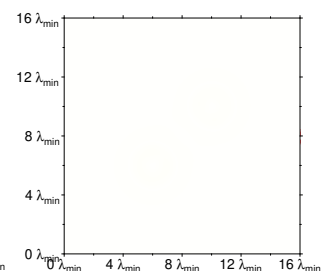

(f)

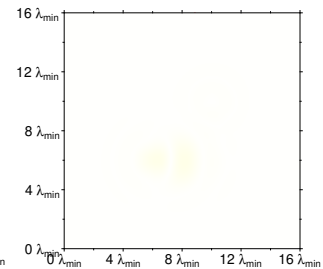

(j)

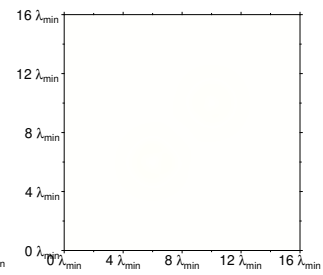

(m)

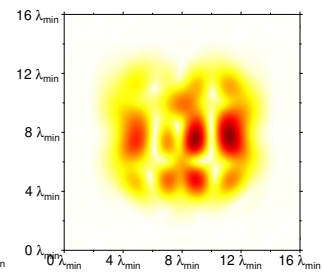

(p)

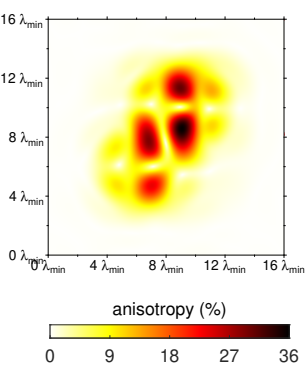

Figure 3. Smooth acoustic FWI tests. On the first line is plotted the $V_{P}$ maps for target model (a) and the homogenized target model (b) as well as a measure of its anisotropy (c) ; In (d), (h) (k) and (n) are plotted $V_{P}$ for the two starting models used here ((d) and (k) are identical as well as (h) and (n)); In (e) and (i) are plotted the $V_{P}^{* \text { iso }}$ maps of the isotropic acoustic inversion homogenized results as well as the corresponding total anisotropy (in (f) and (j) respectively) for the starting models (d) and (h) respectively. In (l) and (o) are plotted the $V_{P}^{* i s o}$ from the anisotropic acoustic inversion homogenized results as well as the corresponding total anisotropy (in (m) and (p) respectively) for the starting models (k) and (n) respectively. 
(a)

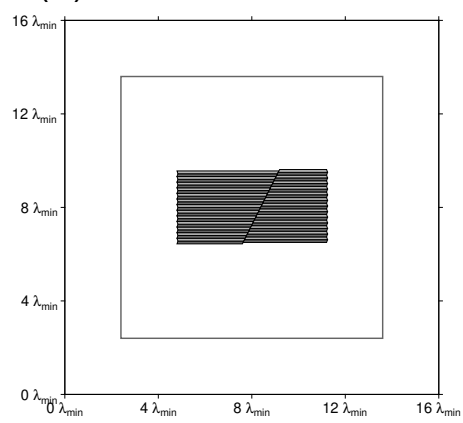

(d)

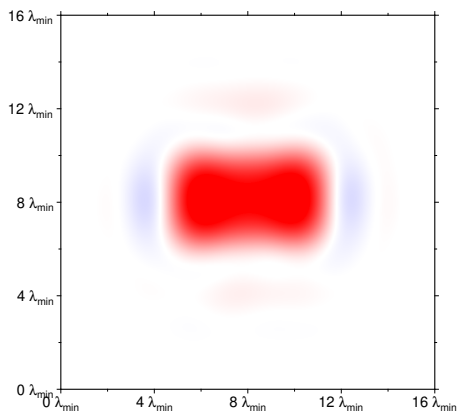

(b)

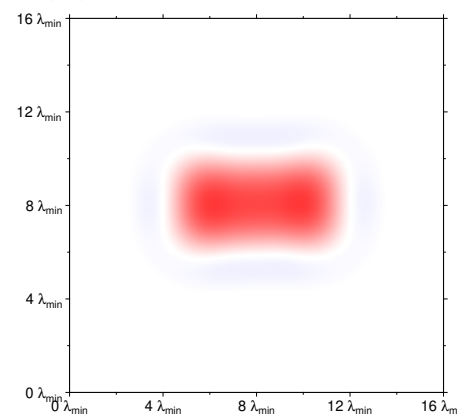

(e)

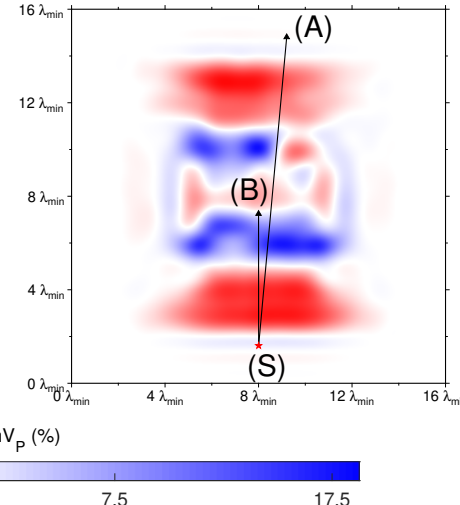

(c)

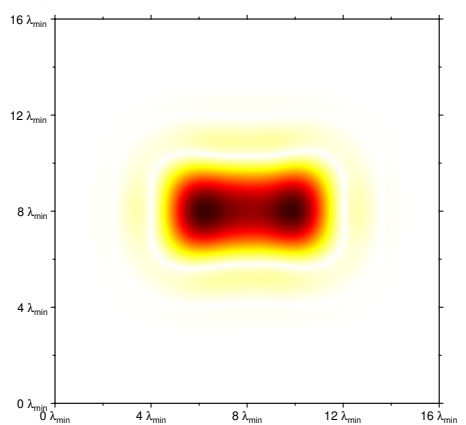

(f)

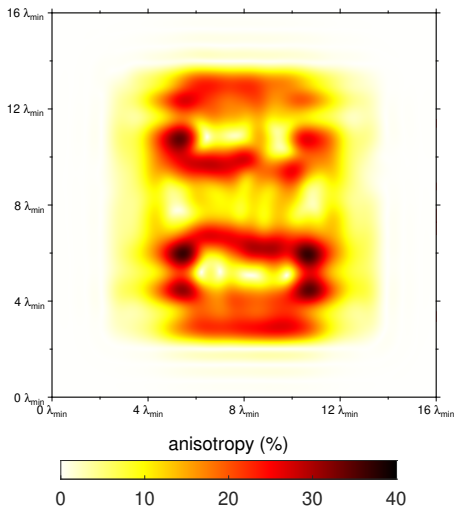

Figure 4. (a): faulted layered target model sketch. This model is acoustic and isotropic. (b) and (c): $V_{P}^{* \text { iso }}$ of the homogenized target model and the corresponding total anisotropy, respectively. (d): $V_{P}^{* \text { iso }}$ homogenized result for an isotropic inversion. (e) and (f): $V_{P}^{* \text { iso }}$ and, respectively, the corresponding total anisotropy for a anisotropic inversion homogenized result. In panel (e), (A), (B) and (S) are the receivers and source positions used in Fig .5 

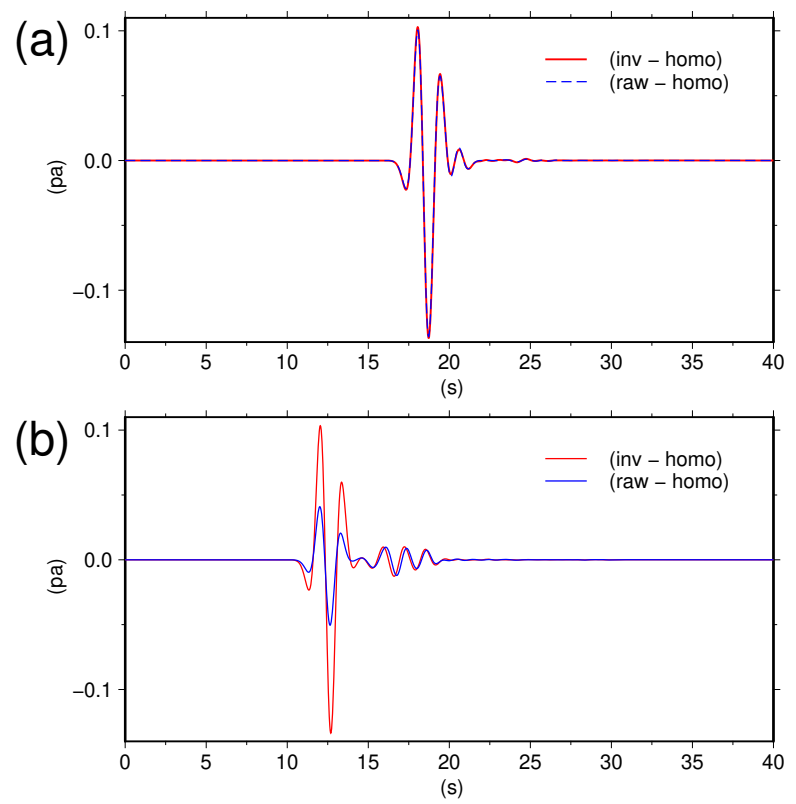

Figure 5. (a) "inv -homo" trace (red line): difference between pressure traces computed in the inverted model and in an homogeneous model for receiver (A) and source (S); "raw-homo" trace (blue dashed): difference between pressure traces computed in the target model and an homogeneous model for receiver (A) and source (S). (b) same as (a) but for receiver (B). The homogeneous model has the same elastic properties as one used outside of the inversion domain. (A), (B) and (S) positions are shown in Fig. 4(e) 